\title{
Searching for the QCD critical point in nuclear collisions
}

\author{
F.K. Diakonos* \\ Physics Department, University of Athens, GR-15771, Greece \\ fdiakono@phys.uoa.gr
}

\section{N.G. Antoniou}

Physics Department, University of Athens, GR-15771, Greece

nantonio@phys.uoa.gr

\section{G. Mavromanolakis}

University of Cambridge, Cavendish Laboratory, High Energy Physics Group, Madingley Road,

Cambridge, CB3 OHE, UK

gmavroma@hep.phy.cam.ac.uk

\begin{abstract}
We present a method of experimental data analysis capable to reveal self-similar transverse momentum density fluctuations in the dipion $\left(\pi^{+}, \pi^{-}\right)$sector produced in $A+A$ collisions. The corresponding fluctuation pattern, measured through the critical index $\phi_{2}$, is associated, through the phenomenon of critical opalescence, with the formation, at the QCD critical point, of a hot isoscalar condensate with transverse fractal geometry. The efficiency of the method is tested in a set of events generated by the Critical Monte-Carlo (CMC) generator. We use the developed algorithm for the experimental data analysis of four different processes $(A=p, C, S i, P b)$ in the NA49 experiment (CERN-SPS) at $\sqrt{s}=17 \mathrm{GeV}$. According to our findings strong self-similar correlations characterize the reconstructed isoscalar sector in the $\mathrm{C}+\mathrm{C}$ and $\mathrm{Si}+\mathrm{Si}$ systems supporting the scenario that the produced fireball in these cases freezes out in the immediate neighbourhood of the QCD critical point.
\end{abstract}

The 3rd edition of the InternationalÂăWorkshop - The Critical Point and Onset of Deconfinement July 3-7 2006

Galileo Galilei Institute, Florence, Italy

\footnotetext{
*Speaker.

${ }^{\dagger}$ The project is co-funded by the European Social Fund and National Resources (EPEAEK II) PYTHAGORAS
} 


\section{Introduction}

The exploration of the QCD phase diagram is one of the central tasks in the new generation of relativistic heavy ion collision experiments providing the unique possibility to test non-perturbative aspects of the fundamental theory of strong interacting matter and to get insight into the well hidden mechanism of hadronization. According to our understanding of strong interactions at finite temperature $T$ and baryonic chemical potential $\mu_{B}$, mainly through recent lattice QCD calculations [1], the quark-hadron phase transition is expected to occur along a critical line in the $\left(\mu_{B}, T\right)$ plane, characterized by first order transitions, and ending at a second order critical point, remnant of the chiral phase transition, in the region of lower baryonic chemical potential and higher temperatures. In fact the chiral symmetry is explicitly broken by small but nonzero quark masses [2] and the benchmark of the critical endpoint, belonging to the $3-D$ Ising universality class [2], is the formation of a hot condensate consisting of almost massless isoscalar $\sigma$-particles. The absense of a characteristic length scale in the $\sigma$-system (very large, practically infinite, correlation length) induces self-similar density fluctuations of the condensate described by the fractal geometry of the formed critical $\sigma$-clusters. The corresponding fractal mass dimension can be determined, using universality class arguments [3], in terms of the isothermal ciritical exponent $\delta$ of the $3-D$ Ising system. As the condensate freezes out, the mass of the sigmas increases beyond the two-pion threshold value and the sigmas decay mainly into pion pairs. Recently it has been proposed that the original self-similar fluctuations of the isoscalar condensate can be experimentally observed by factorial moment analysis in transverse momentum space of the dipion sector, reconstructed from the opposite charged pions produced in a $A+A$ colliding system [4]. The key phenomenon leading to these observable effects is critical opalescence [5] describing the transfer of the real space density fluctuations of critical sigmas to density fluctuations in transverse momentum space. After removing the combinatorial background introduced through the dipion reconstruction the fractal geometry of the remaining fluctuation pattern is revealed and measured in terms of the critical index $\phi_{2}$ [4]. This method of analysis has been succesfully applied to reveal the self-similar fluctuation pattern in a representative ensemble of Monte-Carlo events containing critical sigma sector generated by the CMC algorithm [4]. In the present work we report on a similar analysis applied to SPS-NA49 experimental data for four $A+A$ systems $(A=p, C, S i, P b)$ at maximum available energy. In section 2 we describe briefly the reconstruction algorithm of critical isoscalar flcutuations using as an example to demonstrate its validity a set of CMC generated events. In section 3 we present the preliminary results of a similar analysis applied to NA49 data. Finally in section 4 we summarize our findings and discuss briefly our conclusions.

\section{Reconstruction of critical isoscalar fluctuations}

\subsection{General algorithm}

We follow the scenario when the freeze-out state of the fireball formed during an $A+A$ collision process is characterized by thermodynamic variables $\left(\mu_{B}, T\right)$ in the immediate neighbourhood of (or exactly at) the QCD critical point. The unstable sigma perticles, constituting the condensate, decay dominantly into charged pion pairs and the corresponding momenta are recorded in the detector. The data sets containing the momenta of the produced charged pions in an event-by-event 
basis is the input information in the following analysis. The geometrical characteristics of the critical sigmas in transverse momentum space can be revealed by recombining, within each event, the transverse momenta of the finally produced charged pions according to:

$$
\vec{p}_{\perp, i j}^{\pi \pi}=\vec{p}_{\perp, i}^{+}+\vec{p}_{\perp, j}^{-}
$$

where $\vec{p}_{\perp, i(j)}^{+(-)}$is the transverse momentum vector of the $i$-th ( $j$-th) positive (negative) charged pion in the considered event. Thus, for each event involving charged pions, we construct the matrix $\vec{p}_{\perp, i j}^{\pi \pi}$ containing as entries the transverse momenta of all possible dipions formed by combining a positive and a negative pion from the event. If the multiplicity of charged pions in the $K$-th event is denoted as $n_{\pi^{+}}^{(K)}=n_{\pi^{-}}^{(K)}=n_{\pi, c h .}^{(K)}$. then the above matrix has dimension $n_{\pi, c h .}^{(K)} \times n_{\pi, c h .}^{(K)}$ containing a large number $\left(\left(n_{\pi, c h .}^{(K)}\right)^{2}-n_{\pi, c h}^{(K)}\right)$ of dipions which do not correspond to a real critical sigma-particle in the initial condensate. We will use the term fake sigmas to indicate these dipions in the following. As shown in [4], in order to decrease the relative weight of the fake sigmas in the reconstruction one has to perform the combinatorics (2.1) within a narrow window of dipion invariant mass. To achieve this one uses the filter:

$$
\left(2 m_{\pi}+\varepsilon_{1}\right)^{2} \leq\left(p_{i}^{+}+p_{j}^{-}\right)^{2} \leq\left(2 m_{\pi}+\varepsilon_{2}\right)^{2} \quad \varepsilon_{1}<\varepsilon_{2}
$$

where $p_{i(j)}^{+(-)}$is the four-momentum of the $i$-th ( $j$-th) positive (negative) charged pion in the $K$-th event, $m_{\pi}$ is the pion mass and $\varepsilon_{2}-\varepsilon_{1} \ll 2 m_{\pi}$. The size $\Delta \varepsilon=\varepsilon_{2}-\varepsilon_{1}$ of the invariant mass window in (2.2) has to be chosen appropriately small so that: $\left(n_{\pi, c h .}^{(K)}(\Delta \varepsilon)\right)^{2}-n_{\pi, c h .}^{(K)}(\Delta \varepsilon) \leq n_{\pi, c h .}^{(K)}(\Delta \varepsilon)$ leading to the condition: $n_{\pi, c h .}^{(K)}(\Delta \varepsilon) \leq 2$. At the ensemble level we obtain:

$$
\left\langle n_{\pi, c h .}(\Delta \varepsilon)\right\rangle \leq 2
$$

In practice one has to shift the kinematical window in (2.2) along the invariant mass axis in order to enter into regions with high activity of critical sigmas. Therefore the reconstruction algorithm incorporates two parameters: $\varepsilon_{1}$ and $\Delta \varepsilon$. After fixing the values of these parameters one can construct an ensemble of events involving dipions which respect the conditions (2.1-2.3). The reconstructed events contain both fake as well as real (critical) sigmas. The geometrical characteristics of the real sigmas can be revealed through factorial moment analysis [6] in transverse momentum space of the reconstructed dipions. However the fake sigmas constitute a background in the calculation including unwanted correlations between a pair of fake sigmas or between a fake and a real sigma. As shown in [4] this background can be succesfully removed through a suitable substraction, at the level of factorial moments, using the tool of the hybrid events in order to simulate the unwanted correlations. The construction of the hybrid events implies the appearance of an additional parameter $q$ in the analysis describing the mixing percentage of the original data set. However, as explained in [4], consistency requirements determine $q$ to values close to 1 . The limit $q \rightarrow 1$ means $100 \%$ mixing and in this case the hybrid events coincide with the mixed events. The final observable is the correlator $\Delta F_{2}\left(M, \varepsilon_{1}, \Delta_{\varepsilon}, q\right)$ defined as:

$$
\Delta F_{2}\left(M, \varepsilon_{1}, \Delta \varepsilon, q\right)=F_{2}^{(r)}\left(M, \varepsilon_{1}, \Delta \varepsilon\right)-F_{2}^{(h)}\left(M, \varepsilon_{1}, \Delta \varepsilon, q\right)
$$

where $F_{2}$ is the second factorial moment in transverse momentum space while $M$ is the number of bins in each direction. The superscript $(r)$ indicates the ensemble of events involving reconstructed 
dipions while $(h)$ indicates the corresponding ensemble of hybrid events. According to the basic prediction of Observable Critical $Q C D$ (OCQCD) $\Delta F_{2}$ is expected to have the power-law form: $\Delta F_{2} \sim\left(M^{2}\right)^{\phi_{2}}$ with $\phi_{2} \approx \frac{2}{3}$ [4]. In practice, for a reliable application of the reconstruction algorithm, except of the determination of $\phi_{2}$, one should also ensure the validity of the power-law description for $\Delta F_{2}$ using as control parameters the $\chi^{2}$ per degree of freedom as well as the value of the coefficient of determination $R^{2}$.

\subsection{Reconstruction of the crtical sigma sector in CMC events}

The CMC code generates events incorporating critical sigmas as dictated by OCQCD. A detailed description of the generator as well as the corresponding theoretical basis is presented in [3]. The fractal geometry of the critical sigmas in the CMC generated events is clearly seen in the log-log plot of Fig. 1a showing the second factorial moment in transverse momentum space calculated for an ensemble of 20000 events with mean sigma multiplicity $\left\langle n_{\sigma}\right\rangle \approx 32$. The red line is a linear fit with slope $s_{2} \approx 0.68(01)$ in full agreement with the predictions of OCQCD. The critical sigmas are unstable particles and decay into pions. The momenta of the finally produced pions are determined through the momenta of the initial critical sigmas as well as the corresponding sigma mass distribution $\rho\left(M_{\sigma}\right)$. Measurements of the properties of the sigma particles in medium [7] suggest that for non-zero quark chemical potential the sigma mass distribution should be peaked near the two pion threshold $\left(M_{\sigma} \gtrsim 2 m_{\pi}\right)$. Assuming such a distribution it is straightforward to generate the pion momenta. The critical fluctuation profile is completely lost in the pionic sector due to the kinematical distortion through the $\sigma$-decay. This is shown in Fig. $1 \mathrm{~b}$ displaying the second factorial moment in transverse momentum space of the positive charged pions originating from the decay of the critical sigmas. In order to reveal the initial critical isoscalar flcutuations we apply the reconstruction algorithm described in the previous subsection to the ensemble of events incorporating pions produced from the $\sigma$-decay of the CMC events. The result for the calculation of the correlator $\Delta F_{2}$ using $\varepsilon_{1}=0, \Delta \varepsilon=2 \mathrm{MeV}$ and $q=0.9$ is shown in log-log plot in Fig. 2. The original self-similar fluctuation pattern in the critical isoscalar sector is clearly seen. The linear fit (red line) gives $\phi_{2}=0.66(02)$ in very good agreement with the corresponding result for the critical sigmas presented in Fig. 1a. This analysis suggests that the reconstruction algorithm described in the previous subsection offers a reliable and valuable tool for the search of the QCD critical point through the detection of self-similar isoscalar flcutuations in $A+A$ collision data.

\section{Analysis of $A+A$ NA49 data - preliminary results}

We have used the reconstruction algorithm described in section 2 for the data analysis of four $A+A$ systems measured in NA49 experiment at maximum CERN-SPS energy $E_{L}=158 \mathrm{AGeV}$. After applying several particle identification cuts we end up with the following ensembles: 408708 $p+p$ events, $33689 C+C$ events, $17053 S i+S i$ events and $1487 P b+P b$ events. At the level of total number of tracks the four ensembles are statistically equivalent. A novel issue appearing in the analysis of the real data is the presence of Coulomb correlations between charged pions which are not included in the CMC generated events. Coulomb correlations are peaked for pion pairs with invariant mass approaching the two-pion threshold. Therefore in the invariant mass region just above $2 m_{\pi}$ sigma correlations in the dipion sector, if they exist, are entangled with the 

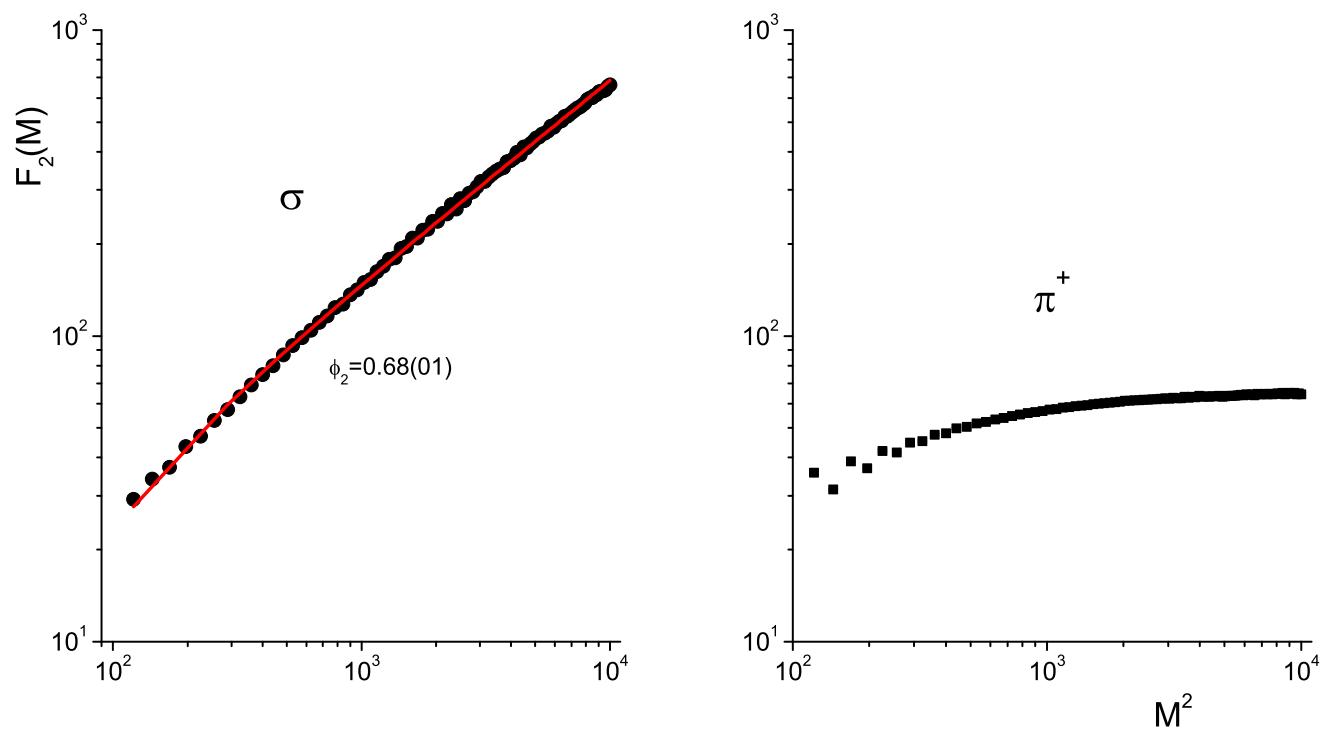

Figure 1: The second factorial moment in transverse momentum space for: (a) 20000 CMC events consisting of critical sigmas and (b) for the positive charged pions resulting from the decay of the critical sigmas analysed in (a).

Coulomb correlations. In order to avoid this entanglement one should choose an appropriate value of $\varepsilon_{1}$ in the filter (2.2). Coulomb correlations are suppressed for pairs of opposite charged pions with $Q_{i n v} \geq 50 \mathrm{MeV}\left(Q_{i n v}=\sqrt{-\left(p^{+}-p^{-}\right)^{2}}\right.$ where $p^{+(-)}$is the four-momentum of the positive (negative) charged pion in the pair). This constraint leads to $\varepsilon_{1} \geq 4 \mathrm{MeV}$. In order to fullfil the requirement (2.3) we have chosen the following sizes for the invariant mass window $\Delta \varepsilon$ in (2.2):

- for the $p+p$ system we use $\Delta \varepsilon=14 \mathrm{MeV}$

- for the $C+C$ system we use $\Delta \varepsilon=2 \mathrm{MeV}$

- for the $S i+S i$ system we use $\Delta \varepsilon=1 \mathrm{MeV}$

- and finally for the $P b+P b$ system we use $\Delta \varepsilon=0.1 \mathrm{MeV}$

In the case of $P b$ the selected value of $\Delta \varepsilon$ is the minimum allowed corresponding to $\Delta Q \approx 5 \mathrm{MeV}$ which is very close to the experimental resolution. Using windows of size $\Delta \varepsilon$ and varying $\varepsilon_{1}$ in the range $[284 \mathrm{MeV}, 320 \mathrm{MeV}]$ we search, for each system, for a region, along the dipion invariant mass axis, with sigma activity, if any. For the $C+C$ and $S i+S i$ systems several such domains exist. The $p+p$ system shows an almost uniform behaviour with respect to translations along the dipion invariant mass axis while for the $P b+P b$ system the limitations discussed above (low statistics, low experimental resolution) do not allow for the determination of an invariant mass interval with enhanced sigma activity. A typical solution in this search, in terms of the size of $\phi_{2}$ as well as the quality of the power-law fit for $\Delta F_{2}$ measured through $\chi^{2} / d o f$ and $R^{2}$, is shown in Fig. 3. In Fig. 4 


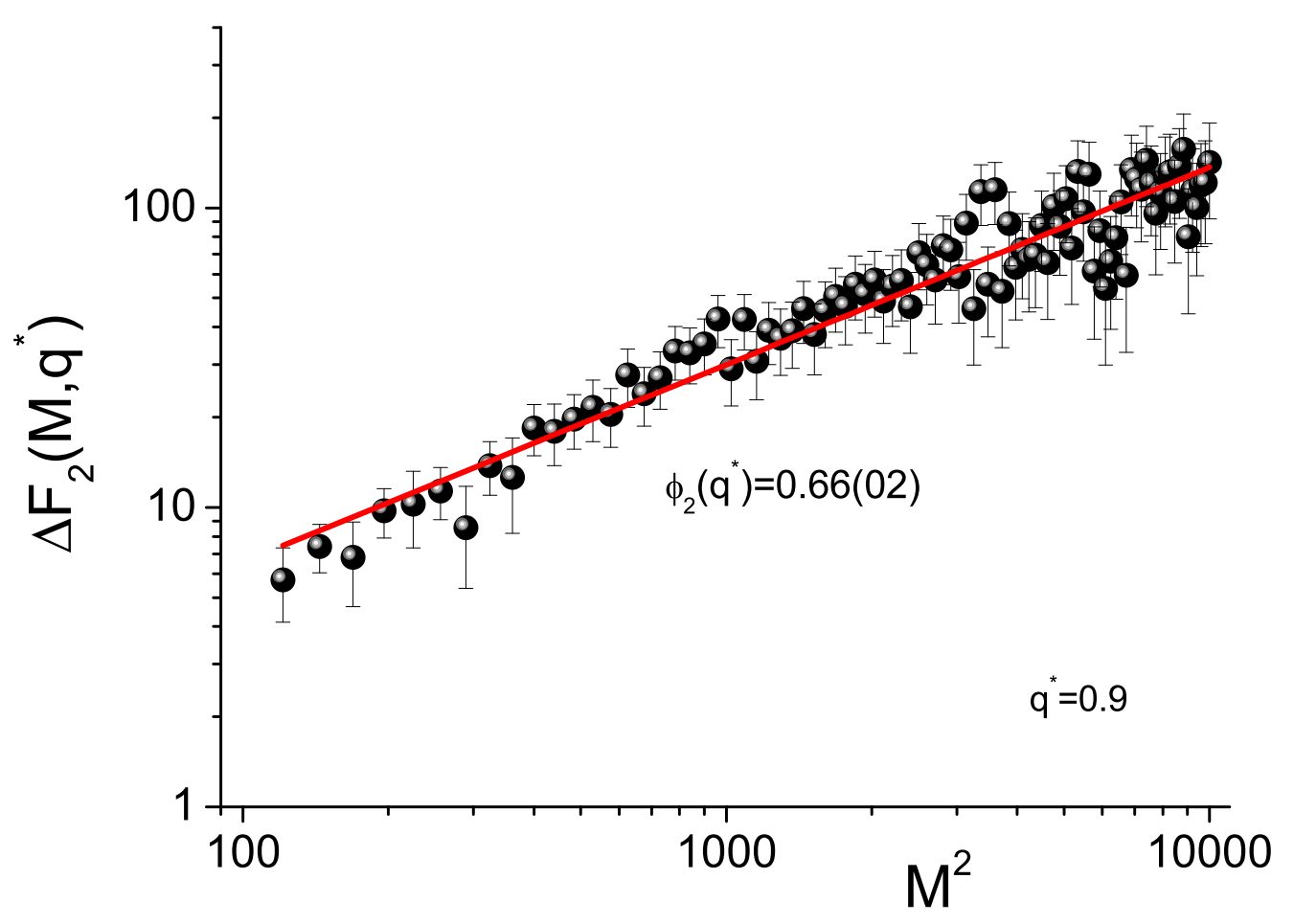

Figure 2: The second factorial moment in transverse momentum space for dipions reconstructed from opposite charged pions produced through the decay of the critical sigmas.

we show the $\phi_{2}$ dependence on the system size $A$ resulting from the solution displayed in Fig. 3. The horizontal line presents the OCQCD prediction for $\phi_{2}\left(\phi_{2, c}=\frac{2}{3}\right)$. It is clear that the relatively small systems $C$ and $S i$ approach the critical QCD prediction while the corresponding fit quality is in acceptable range $\left(\chi^{2} / d o f<1\right.$ and $\left.R^{2}>0.6\right)$. The latter is not true for the $P b$ system possibly due to the fact that $\Delta \varepsilon$ for this system should be further decreased. However with the existing experimental resolution this is not possible. Therefore the obtained $\phi_{2}$ value for $P b+P b$ has to be considered only as a lower limit. Our analysis suggests that the $S i$ system freezes out very close to the critical point. It is interesting to compare the $S i$ result with that of the CMC events with a similar charged pion multiplicity. In addition one could also apply the reconstruction algorithm to simulated $\mathrm{Si}+\mathrm{Si}$ events using a conventional Monte-Carlo generator like HIJING [8]. This analysis is performed and the corresponding results are shown in Fig. 5. It is obvious the similarity between the CMC and the NA49 behaviour. Contrary to that, in the HIJING events no such power-law dependence of $\Delta F_{2}$ is observed. This finding supports further the scenario that the correlations in the $\sigma$-mode generated in the $S i+S i$ system are unconventional and can be associated with the approach to the QCD critical point. 

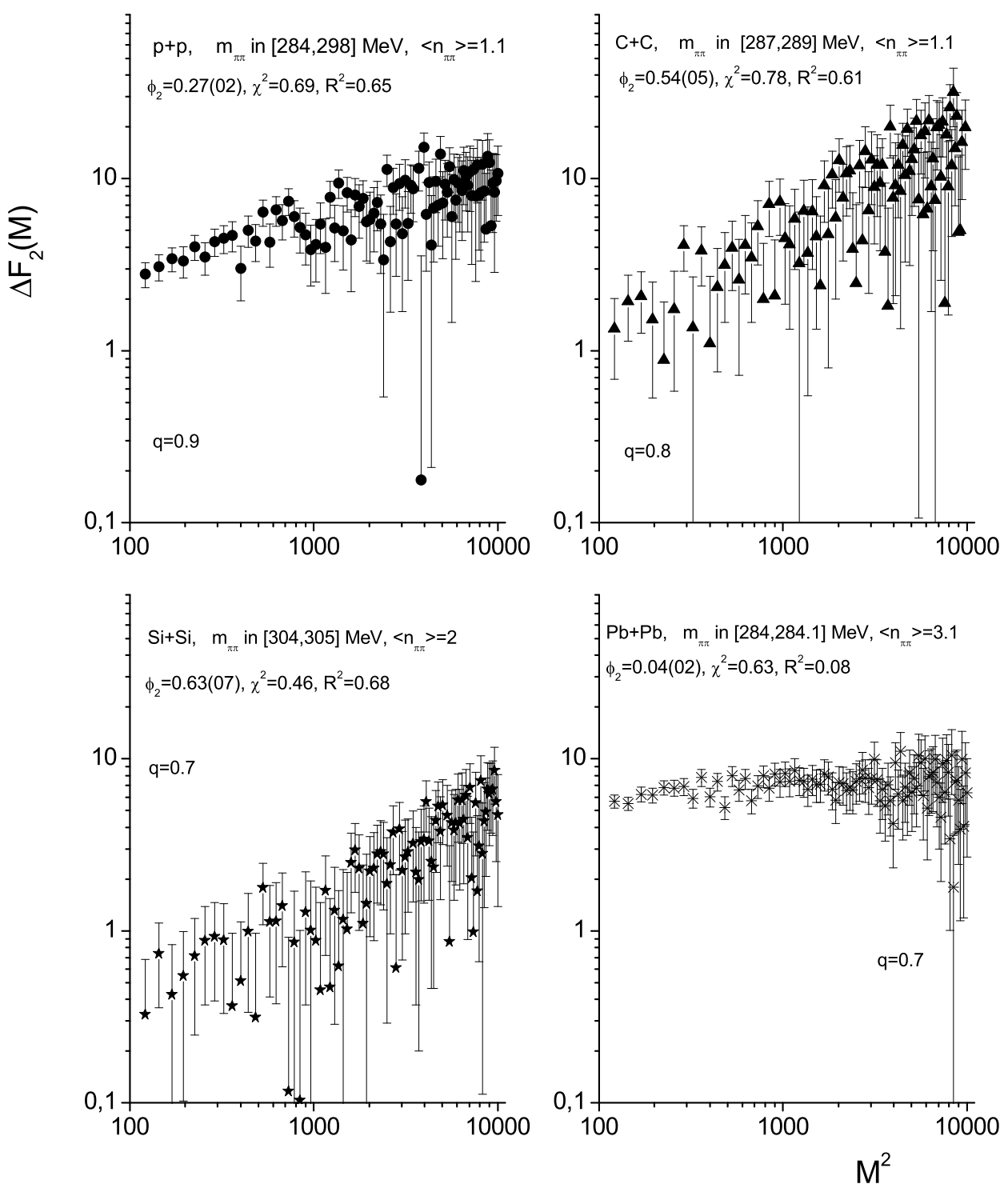

Figure 3: A typical solution for the correlator $\Delta F_{2}$, in a search for kinematical domains with enhanced sigma activity, using in the analysis the data of the four considered $A+A$ systems. 


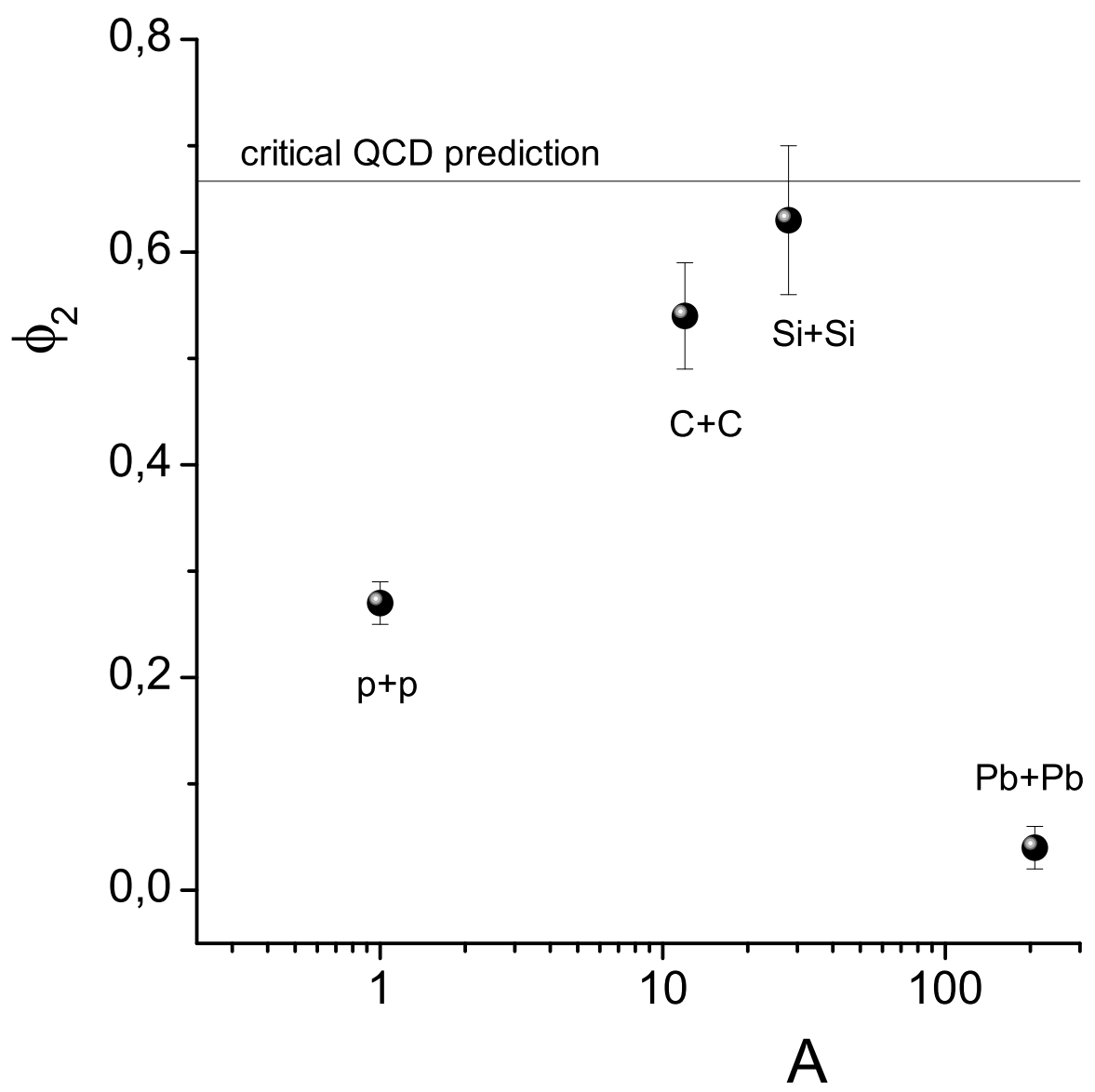

Figure 4: The critical index $\phi_{2}$, determined through the solution shown in Fig. 3, as a function of the size $A$ of the corresponding nuclei.

\section{Concluding remarks}

A method of analysis to look for isoscalar critical fluctuations in $A+A$ data is developed. Within this method the disentanglement between Coulomb $\left(\pi^{+}, \pi^{-}\right)$correlations and sigma correlations in the reconstructed dipion sector is possible. Applying this method to NA49 data a clear signature for the presence of unconventional correlations in the $S i+S i$ and $C+C$ system at $158 \mathrm{AGeV}$ is obtained. The size of these correlations, especially for the $\mathrm{Si}+\mathrm{Si}$ system, measured in terms of the critical index $\phi_{2}$ is close to the theoretical expectation for an $A+A$ collision system freezing out close to the QCD critical point. The errors in the above analysis are relatively large and higher statistics (unfortunately not available at the present stage) is needed for a more accurate 

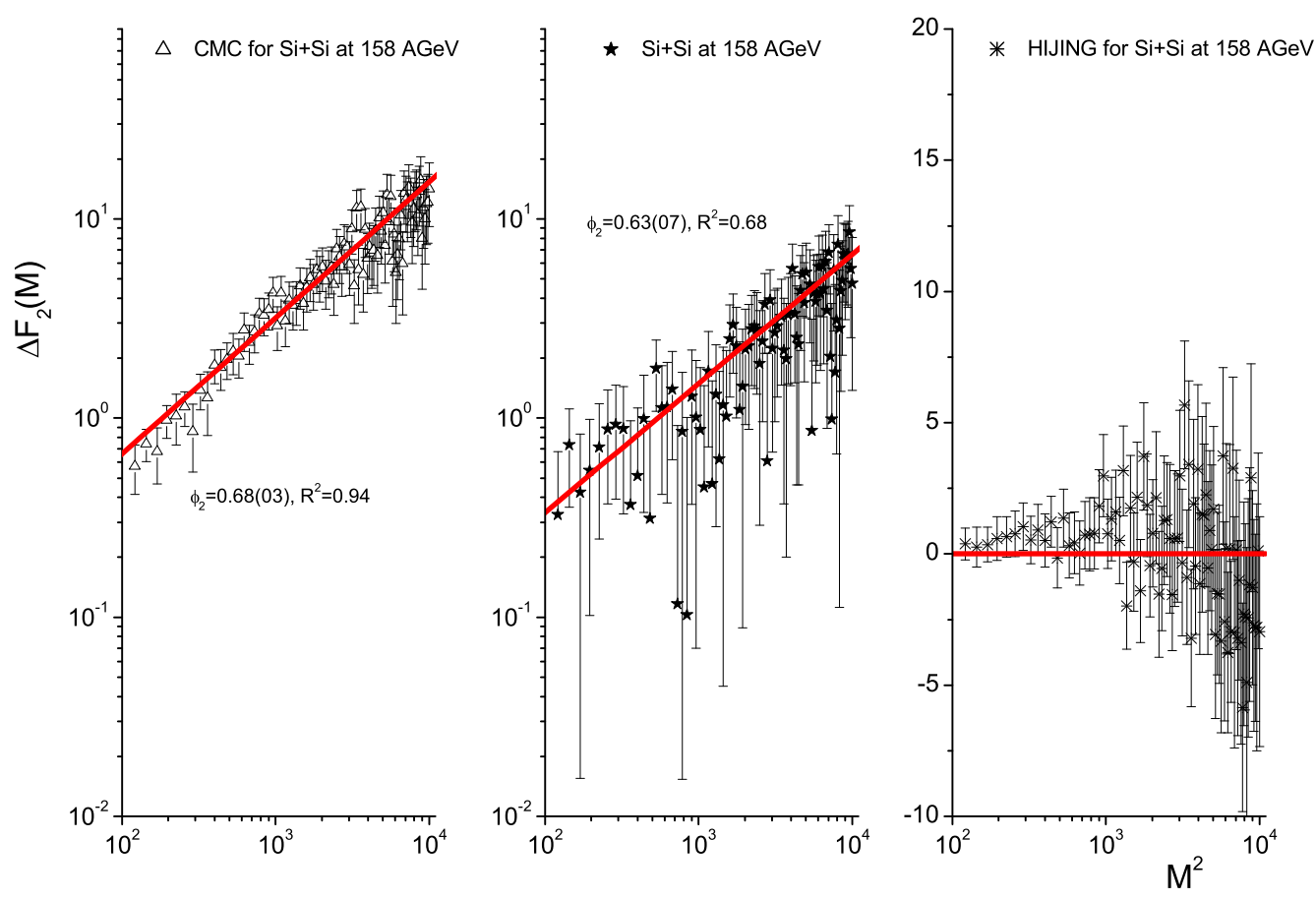

Figure 5: $\Delta F_{2}$ in transverse momentum space for: (a) $20000 \mathrm{CMC}$ events with mean charged pion multiplicity $\approx 16$, (b) $S i+S i$-data of the NA49 experiment at $E_{L}=158 \mathrm{GeV} / n$ and (c) 15000 HIJING events simulating the $S i+S i$ system.

calculation of $\phi_{2}$. Our results support the need for an extented exploration of the QCD phase diagram in the neighbourhood of the freezing-out conditions of the lighter systems at energies close to the maximum SPS energy $158 \mathrm{AGeV}$.

\section{References}

[1] Z. Fodor, S. D. Katz, J. High Energy Phys. 0404 (2004) 050; Z. Fodor, S. D. Katz, J. High Energy Phys. 03 (2002) 014; C. R. Allton, S. Ejiri, S. J. Hands, O. Kaczmarek, F. Karsch, E. Laermann, C. Schmidt, Nucl. Phys. Proc. Suppl. B129, 614 (2004); Phys. Rev. D68, 014507 (2003); R. V. Gavai, S. Gupta, Phys. Rev. D71, 114014 (2005).

[2] F. Wilczek, hep-ph/0003183; M. A. Halasz, A. D. Jackson, R. E. Shrock, M. A. Stephanov and J. J. M. Verbaarschot, Phys. Rev. D58, 096007 (1998); M. Stephanov, K. Rajagopal, E. Shuryak, Phys. Rev. Lett. 81, 4816 (1998); M.A. Stephanov, Prog. Theor. Phys. Suppl. 153, 139 (2004); Int. J. Mod. Phys. A20, 4387 (2005); hep-ph/0402115.

[3] N. G. Antoniou, Y. F. Contoyiannis, F. K. Diakonos, A. I. Karanikas, C. N. Ktorides, Nucl. Phys. A693, 799 (2001).

[4] N. G. Antoniou, Y. F. Contoyiannis, F. K. Diakonos, G. Mavromanolakis, Nucl. Phys. A761, 149 (2005). 
[5] H. E. Stanley: Introduction to Phase Transitions and Critical Phenomena, Oxford University Press, 1987; P. M. Chaikin, T. C. Lubensky: Principles of condensed matter physics, Cambridge University Press, 1997.

[6] A. Bialas and R. Peschanski, Nucl. Phys. B273, 703 (1986); Nucl. Phys. B308, 857 (1988).

[7] CHAOS Collaboration, N. Grion et al, Nucl. Phys. A763, 80 (2005).

[8] M. Gyulassy and X.N. Wang, Comp. Phys. Comm. 83, 307 (1994). 